\title{
PPAS: Programmable Power Analysis Method for Analyzing and Optimizing Power Consumption in Smartphone
}

\author{
Rashida Hasan \\ Dept. of Computer Science \& \\ Engineering \\ University of Dhaka \\ Bangladesh
}

\author{
Feroz Jahangir Rana \\ Dept. of Computer Science \& \\ Engineering \\ University of Dhaka \\ Bangladesh
}

\author{
Jainal Uddin \\ Dept. of Computer Science \& \\ Engineering \\ University of Dhaka \\ Bangladesh
}

\begin{abstract}
New and innovative applications for mobile consumerelectronic devices, especially for Smartphones are developing rapidly, advances in battery technology have not kept pace with rapidly growing energy demands. Since, the batteries are limited in size and therefore capacity, power consumption is a critical concern for battery driven Smartphone and this implies that managing energy well is paramount in such device. The core need for good energy management requires a better understanding of where and how the energy is used: how much of the system's energy is consumed by which parts of the system and under what circumstances. This paper focuses on understanding the power consumption of major hardware components and their usage on different scenarios. We present a detail analysis of the power consumption of iPhone and develop a method PPAS-programmable power analysis method to analyze power consumption in different scenarios. This work identifies the most promising areas to focus on for further improvements of power management as well as implement some effective techniques to optimize power consumption.
\end{abstract}

\section{Keywords}

Smartphone, power consumption, iPhone, iOS

\section{INTRODUCTION}

A Smartphone or smart phone is a mobile phone built on a mobile operating system, with more advanced computing capability and connectivity than a feature phone [1]. These devices promise to offer a large spectrum of applications including communication, planning, entertainment, web browsing, Wi-Fi, multimedia applications such as streaming video players, games, image and video capturing tools, location-aware mobile applications, location-based tracking applications and more. The Smartphone usage can be characterized into four key dimensions including user interactions; application usage, network traffic and energy drain [2]

The complexity and capabilities of these devices continues to grow at an amazing pace, but battery capacity is severely restricted due to constrain on size and weight of the device. Therefore, the power consumption has emerged as a key issue of the energy management of portables. Smartphone manufacturers have now much attention on the optimization of power consumption. Due to the lack of proper understanding of power usage in Smartphone, a lot of applications have not developed in perfect ways; therefore power is draining rapidly when the applications are running. This implies that energy efficiency of these devices is very important to their usability and needs to optimize the usage of power.

Effective and efficient energy management requires good understanding of where and how the energy is used, how much energy is consumed by which parts of the system and under what circumstances.

This paper attempts to answer these questions and provide a good analysis of the uses of power by different functionalities. We collect power consumption data using software which is a better solution for latest devices. Because it is very difficult to take physical power measurements at the component level on a piece of real hardware due to the integration of hardware components into a single chips or board. Some effective and optimized techniques are also discussed with comparison data which will help all the Smartphone application developers to develop applications with optimized ways to save powers.

The rest of the paper is organized as follows. Section 2 presents the related work regarding power consumption in Smartphones. Section 3 provides a brief overview of Smartphone architecture and the operating systems used in Smartphones. In section 4, we present the data collection methodology. Section 5 presents the experimental results with effective techniques to optimize power consumption. Finally, we conclude our work on section 6.

\section{RELATED WORK}

Recently Smartphones have received significant attention from researchers. The advancement and growth of Smartphone depends on the proper usage of power and performance. Many researchers mentioned their own ways to save energy in Smartphones.

Caroll presented a detailed analysis of the power consumption of an old Smartphone device named Openmoko [3]. Power consumption is analyzed by taking physical power measurements at the component level on a piece of real hardware. Sense resistors are inserted on the power supply rails of the major hardware components which were possible because the selected device has been designed with placeholders for sense resistors. But now it is very difficult to take physical power measurement at the component level on a piece of real hardware because most of the components of hardware are integrated into a single chip or board.

Fangwei [4] and Igor [5] present a framework for energy monitoring that includes hardware set up, along with current observations from energy profiling of a mobile phone. However, they focused on understanding the energy consumption of physical activity monitoring applications and 
did not provide energy consumption monitoring in a developers perspective. Both of these researches are done on Android Smartphone.

\section{SMARTPHONE ARCHITECTURE}

This section describes the Smartphone architecture and their OS platform. The configuration of the hardware platform currently used in a Smartphone is shown schematically in figure 1 .

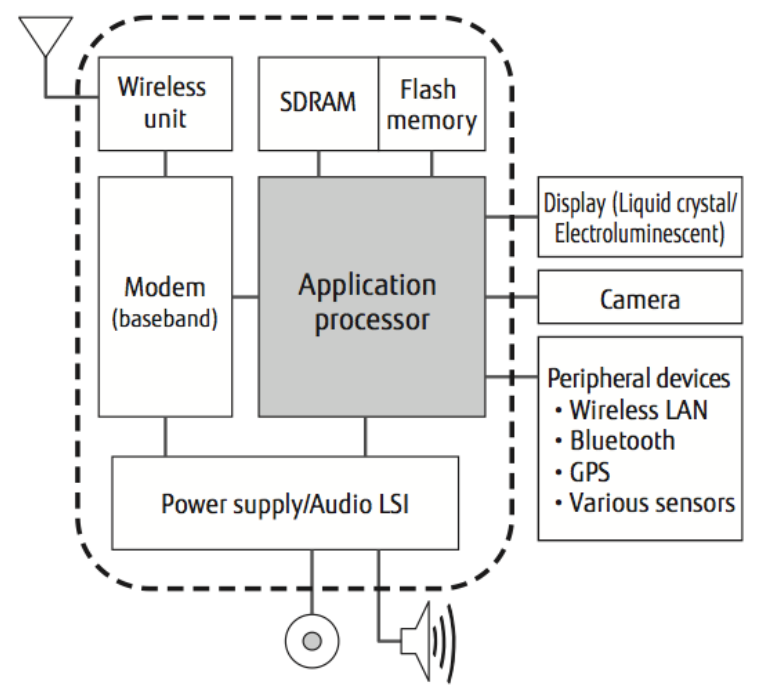

Fig 1: Smartphone hardware platform [6]

The platform is composed of an application processor, a modem (baseband), a wire-less unit, a power supply, an audio LSI, synchronous dynamic random access memory (SDRAM) and flash memory.

The application processor is composed of a CPU, a graphics processing unit (GPU) and controllers for its memory, peripheral devices and display. These are the key components for attaining the high performance and functionality needed for a Smartphone. The demand for increased performance has driven the evolution of ARM CPU which is 32-bit RISC processor featuring highly optimized architecture, with few transistors and extremely low power consumption.

A graphic processing unit (GPU) is a specialized electronic circuit designed to rapidly manipulate and alter memory to accelerate the creation of images in a frame buffer intended for output to a display. Modern GPUs are very efficient at manipulating computer graphics and their highly parallel structure makes them more effective than general-purpose CPUs.

Smartphone operating systems combine the features of a personal computer operating system with other features including a touch screen, cellular, Bluetooth, Wi-Fi, GPS mobile navigation, camera, video camera, speech recognition, voice recorder, music player, near field communication and more. The most common Smartphone operating systems are iOS, Android, Blackberry, Symbian and Windows phone.

\section{PPAS: DATA COLLECTION METHODOLOGY}

Presently, it is very difficult to take physical power measurement at the component level on a piece of real hardware because most of the components of hardware are integrated into a single chip or board. So we cannot use the power calculation equation $P=V t$ easily. This research presents PPAS (Programmable Power Analysis Method in Smartphone) to analyze the energy consumption of the application on Smartphone. iOS is considered for developing PPAS method and all data collection and experimental results are made based on the output of the applications of iPhone. PPAS technique for data collection is applicable to other platforms like android, Windows phone etc. Table 1 shows the iPhone $4 \mathrm{~s}$ and iPhone 5 specification [7], [8].

Table 1. iPhone $4 \mathrm{~s}$ and iPhone 5 specification

\begin{tabular}{|c|c|c|}
\hline Design & Apple iPhone 4s & Apple iPhone 5 \\
\hline Hardware & Apple A5 & Apple A6 \\
\hline System chip & $\begin{array}{c}\text { Dual core, } \\
\text { 800MHz, ARM } \\
\text { Cortex-A9 }\end{array}$ & $\begin{array}{c}\text { Dual core, 1300 } \\
\text { MHz, Apple Swift }\end{array}$ \\
\hline Processor & $\begin{array}{c}\text { PowerVR } \\
\text { SGX543 MP2 }\end{array}$ & $\begin{array}{c}\text { PowerVR } \\
\text { SGX543 MP2 }\end{array}$ \\
\hline $\begin{array}{c}\text { Graphics } \\
\text { processor }\end{array}$ & $\begin{array}{c}512 \mathrm{MB} \\
\text { RAM/DDR2 (800 } \\
\text { MHz) }\end{array}$ & \\
\hline $\begin{array}{c}\text { System } \\
\text { memory }\end{array}$ & $\begin{array}{c}\text { GB RBMDR2 } \\
\text { Built-in } \\
\text { storage }\end{array}$ & 16 GB \\
\hline Display & & 4 inches \\
\hline Physical size & 3.5 inches & $326 \mathrm{p} \mathrm{ppi}$ \\
\hline Resolution & $640 \times 940$ pixels & $640 \times 1136$ pixels \\
\hline Pixel density & 326 ppi & \\
\hline \multicolumn{2}{|c|}{}
\end{tabular}

To profiling the energy consumption in iPhone, PPAS method first establishes the baseline power state of the device which is a state when no applications are running, even no task in background. To get more accurate power consumption data, it considers one important feature of iOS that is airplane mode which disables the wireless features of iPhone including cellular (voice and data), Wi-Fi, Bluetooth, GPS and location services. During data collection, PPAS also take into account the power consumption of display based on some functionality. Then it runs specific task for specific components for long time to get data. The real power consumption data is obtained by comparing the new data with old data.

While taking sample, the phone is kept busy only with that operation as we focus on power consumption or discharge rate. For example, when collecting sample for browsing using GPRS (2G), Wi-Fi, BT, GPS, Auto sync are kept off. In addition, it keeps minimum number of tasks running and killed most of the task of the device before taking sample data. Figure 2 shows the algorithmic description of PPAS.

\section{Algorithm: PPAS}

$[\mathrm{dr}[\mathrm{B}]$ is the discharge rate in minutes at brightness $\mathrm{B}]$

1. Establish base line power state of the device

2. IF operation does not need network service

SET device into airplane mode 
3. Collect baseline power consumption data with some fixed interval periodically

4. Run specific task for specific component for long time

5. Consider brightness in calculation

$$
\begin{aligned}
& \text { IF backlight is on } \\
& \text { Collect data with } 100 \% \text { and } \\
& 15 \% \text { fixed brightness } \\
& d r[B]=\frac{d r 100-d r 15}{100-15} *(B-15)+d r 15
\end{aligned}
$$

6. Again collect power consumption data with a fix interval periodically

7. Compare this data with baseline data to get actual power consumption data

\section{Fig 2: Algorithmic description of PPAS}

The major functionalities that was taken into account for data collection includes browsing, video playback, audio playback, Bluetooth, talk time, voice call, navigation, OpenGL drawing, camera, standby and airplane mode.

\subsection{Browsing}

A web browser is a software application for retrieving, presenting and traversing information resources on the World Wide Web. PPAS calculates the power consumption for a web browsing workload using both GPRS and Wi-Fi. It sets some fixed popular links which is called repeatedly to make the application busy with only browsing. It considers backlight with brightness $15 \%$ and $100 \%$ during collecting power consumption data of browsing because one has to use backlight during browsing. Table 2 shows the collected average data for browsing on iPhone $4 \mathrm{~S}$ and iPhone 5 .

Table 2. Power consumed for browsing on iPhone $4 \mathrm{~S}$ and iPhone 5

\begin{tabular}{|l|c|c|}
\hline Discharge/min & iPhone 4S & iPhone 5 \\
\hline Browsing 2G (15\%) & 0.195501 & 0.194378 \\
\hline Browsing 2G (100\%) & 0.216198 & 0.209198 \\
\hline Browsing 3G (15\%) & 0.308641 & 0.221021 \\
\hline Browsing 3G (100\%) & 0.340136 & 0.234741 \\
\hline Browsing Wi-Fi (15\%) & 0.205761 & 0.181159 \\
\hline Browsing Wi-Fi (100\%) & 0.219298 & 0.193798 \\
\hline
\end{tabular}

\subsection{Video Playback}

Power consumption of video playback is very high comparing to other features. PPAS considers backlight with brightness $15 \%$ and $100 \%$ during collecting power consumption data of playing video. For experimental set up a long duration video is played and take sample with fixed interval. Hardware and software decoders are used to play video which has a significant impact on power discharge rate. Software decoding is flexible and support many file types than hardware decoding. Since software decoder performs its video processing task on CPU so it uses more power than hardware decoder. FFmpeg library is used for software decoder to measure power consumption. Table 3 shows the collected average data for video playback using hardware decoder and table 4 shows the power consumption data for video playback using software decoder.

\subsection{Audio Playback}

The measurements for audio playback are taken with backlight off because typically someone listens to music while carrying the phone in their pocket. The samples are collected by playing a long duration audio music. While taking samples, all other applications are stopped from running in background. The power consumption data for audio playback is shown in table 5 .

Table 3. Power consumption of video playback using hardware decoder

\begin{tabular}{|c|c|c|}
\hline Discharge/min & iPhone 4S & iPhone 5 \\
\hline Video Playback (15\%) & 0.202125 & 0.199601 \\
\hline Video Playback (100\%) & 0.234741 & 0.229885 \\
\hline Airplane ON (15\%) & 0.181159 & 0.178826 \\
\hline Airplane ON (100\%) & 0.204498 & 0.198412 \\
\hline
\end{tabular}

Table 4. Power consumption of video playback using software decoder

\begin{tabular}{|c|l|l|}
\hline Discharge/min & iPhone 4S & iPhone 5 \\
\hline Video Playback (15\%) & 0.262647 & 0.260824 \\
\hline Video Playback (100\%) & 0.305250 & 0.303582 \\
\hline Airplane ON (15\%) & 0.233764 & 0.210970 \\
\hline Airplane ON (100\%) & 0.259201 & 0.256016 \\
\hline
\end{tabular}

Table 5. Power consumed for audio playback on iPhone $4 \mathrm{~S}$ and iPhone 5

\begin{tabular}{|c|c|c|}
\hline Discharge/min & iPhone 4S & iPhone 5 \\
\hline Audio Playback & 0.046296 & 0.045045 \\
\hline Airplane ON & 0.045167 & 0.043630 \\
\hline
\end{tabular}

Table 6. Power consumed for Bluetooth on iPhone 4S and iPhone 5

\begin{tabular}{|c|c|c|}
\hline Discharge/min & iPhone 4S & iPhone 5 \\
\hline Bluetooth & 0.081699 & 0.079744 \\
\hline Bluetooth & 0.091074 & 0.087260 \\
\hline
\end{tabular}

Table 7. Power consumed for talktime on iPhone $4 S$ and iPhone 5

\begin{tabular}{|c|c|c|}
\hline Discharge/min & iPhone 4S & iPhone 5 \\
\hline Talktime 2G & 0.126263 & 0.135501 \\
\hline Talktime 3G & 0.219298 & 0.209643 \\
\hline
\end{tabular}

\subsection{Bluetooth}

iPhone Bluetooth have some restriction like it can't transfer file from iPhone device to other types of Smartphones. So an external accessory is used to transfer data between them with a fixed data rate of $55 \mathrm{kbps}$. The measurements are taken with backlight off and stop all other application running in the background. The power consumption average data for Bluetooth is presented in table 6 .

\subsection{Talktime}

The main purpose of a phone is that it can make and receive phone calls over a radio link while moving around a wide geographic area. It does so by connecting to a cellular network provided by a mobile phone operator allowing access 
to the public telephone network. The power consumption average data for talktime is shown in table 7 .

Table 8. Power consumption of video voice call

\begin{tabular}{|c|c|c|}
\hline Discharge/min & $\begin{array}{c}\text { iPhone } \\
\text { 4S }\end{array}$ & iPhone 5 \\
\hline Skype Video Call 3G (15\%) & 0.375000 & 0.311111 \\
\hline Skype Video Call 3G (100\%) & 0.533333 & 0.375000 \\
\hline Skype Video Call Wi-Fi (15\%) & 0.20000 & 0.257143 \\
\hline Skype Video Call Wi-Fi (100\%) & 0.366667 & 0.300000 \\
\hline
\end{tabular}

\subsection{Voice Call (Skype)}

The measurement of power consumption of video voice call is taken using Wi-Fi and GPRS network while considering backlight turn on. But in audio voice call, backlight is skipped since it is not necessary. The collected average data for video voice call and audio voice call is shown in table 8 and table 9 .

Table 9. Power consumption of audio voice call

\begin{tabular}{|c|c|c|}
\hline Discharge/min & iPhone 4S & iPhone 5 \\
\hline Skype Audio Call 3G & 0.135501 & 0.126198 \\
\hline Skype Audio Call Wi-Fi & 0.104872 & 0.098730 \\
\hline
\end{tabular}

Table 10. Power consumption of Navigation

\begin{tabular}{|c|c|c|}
\hline Discharge/min & iPhone 4S & iPhone 5 \\
\hline Navigation (15\%) & 0.375000 & 0.311111 \\
\hline Navigation (100\%) & 0.403213 & 0.375000 \\
\hline
\end{tabular}

Table 11. Power consumption of OpenGL drawing

\begin{tabular}{|c|c|c|}
\hline Discharge/min & iPhone 4S & iPhone 5 \\
\hline OpenGL (15\%) & 0.231481 & 0.210970 \\
\hline OpenGL (100\%) & 0.252321 & 0.245098 \\
\hline Airplane mode ON (15\%) & 0.213675 & 0.200803 \\
\hline Airplane mode ON (100\%) & 0.234741 & 0.2283105 \\
\hline
\end{tabular}

Table 12. Power consumption of camera

\begin{tabular}{|l|c|c|}
\hline \multicolumn{1}{|c|}{ Discharge/min } & iPhone 4S & iPhone 5 \\
\hline Camera (15\%) & 0.303012 & 0.292079 \\
\hline Camera (15\%) & 0.370371 & 0.346500 \\
\hline Airplane ON & 0.283224 & 0.264131 \\
\hline
\end{tabular}

Table 13. Power consumption of standby

\begin{tabular}{|c|c|c|}
\hline Discharge/min & iPhone 4S & iPhone 5 \\
\hline Cellular 2G & 0.006768 & 0.005453 \\
\hline Cellular 3G & 0.009259 & 0.008333 \\
\hline
\end{tabular}

\subsection{Navigation}

iPhone has a GPS unit that allows the sending and receiving of signals to and from satellites to determine exact location, which is integral for some apps to work, for example, mapbased apps like Google Maps or to check-in on Facebook. Some of these apps may continue to send and receive signals during running in the background which takes a lot of our battery to continuously do that even if we aren't aware of it. Therefore, all those apps are stopped if we really don't need them. The backlight with brightness $15 \%$ and $100 \%$ is taken into account when dealing with maps or locations. Table 10 presents the collected average data of power consumption for navigation.

\subsection{OpenGl Drawing}

To measure the power consumption of OpenGL, an application is made that uses OpenGL framework to draw some objects on display. The app has run for long time to collect power consumption data and stopped all other applications during running in the background. Table 11 shows the collected average data of power consumption for OpenGL drawing.

\subsection{Camera}

Table 12 shows the power consumption when using camera. The backlight is considered during collecting power consumption data of camera and stopped all the applications running in the background

\subsection{Standby}

To measure the power consumption of standby, all other applications are stopped running in the background and keep airplane mode OFF because Smartphone is used in this state. For verification, we compare our collected data with the provided technical specification of the device. Table 13 presents the collected average data for standby on iPhone $4 \mathrm{~s}$ and iPhone 5.

\subsection{Airplane Mode}

To measure the power consumption in airplane mode, all other application are stopped running in the background. The setting of iOS is checked to verify that the airplane mode is ON. Table 14 shows the average power consumption data for airplane mode $\mathrm{ON}$.

\section{Table 14. Power consumption data for Airplane mode}

\begin{tabular}{|c|c|c|}
\hline Discharge/min & iPhone 4S & iPhone 5 \\
\hline Airplane ON & 0.005376 & 0.004761 \\
\hline
\end{tabular}

\section{RESULTS}

In this section, some effective techniques are discussed for optimizing power consumption in iPhone. These techniques are also applicable to other platforms such as android, Windows phone etc. The results are also presented for the purpose of demonstrating the efficiency and effectiveness of proposed techniques. The superiority of proposed optimized techniques has been demonstrated by comparing it with normal way of power consumption. To develop PPAS, we use Macbook Pro: core i5 PC and xCode 4.5 as IDE (Integrated development Environment) and Xcode instrumental tools are used for performance analysis and memory issues.

\subsection{Wi-Fi with GPS System}

After analyzing power consumption data of Wi-Fi with various scenarios, it is found that discovery time and trying to connect time of Wi-Fi in Smartphone plays an impact on power consumption. Most of the times we forget to switch OFF our Wi-Fi option on Smartphone when it's not necessary. So it tries to discover and connect with Bluetooth devices several times periodically which consumes unnecessary power and drains out battery. The solution of this problem is to automatically turn ON or OFF Bluetooth option of Smartphone using GPS when it's not necessary. PPAS method set up the environment with 3 defined zones. 
Therefore, if we go outside of the range of areas, GPS notify the Smartphone to turn OFF Wi-Fi automatically so that unnecessary use of Wi-Fi will not drain the power of battery.

To compare proposed approach with normal usage approach, we installed app and run for a long time with switching among different zones. The comparison of these two approaches is shown in figure 3. It is obvious from the figure that our optimized way using Wi-Fi takes less power than the normal way and demonstrates the efficiency of our approach.

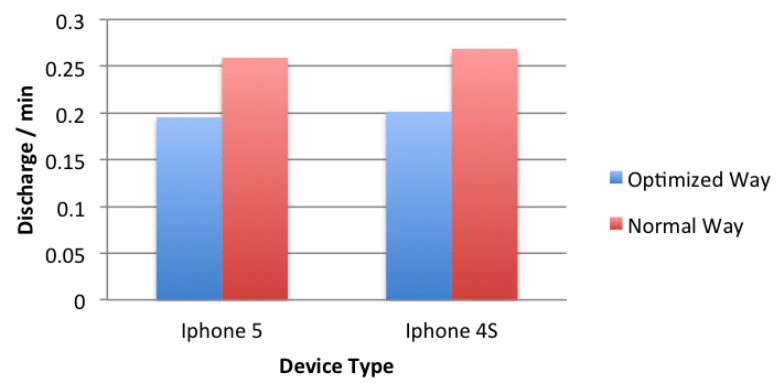

Fig 3: Usage of Wi-Fi with GPS system

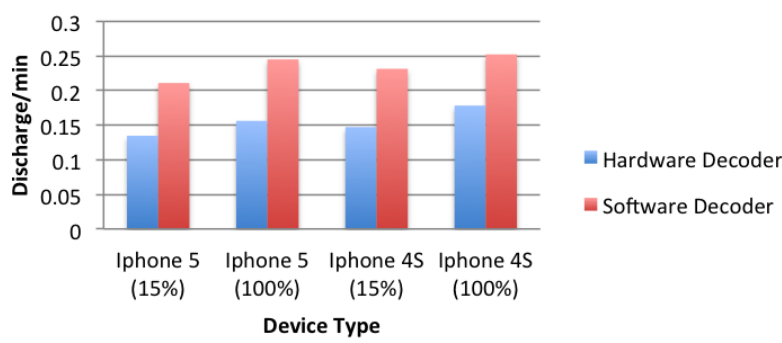

Fig 4: Video play comparison: hardware vs. software decoder

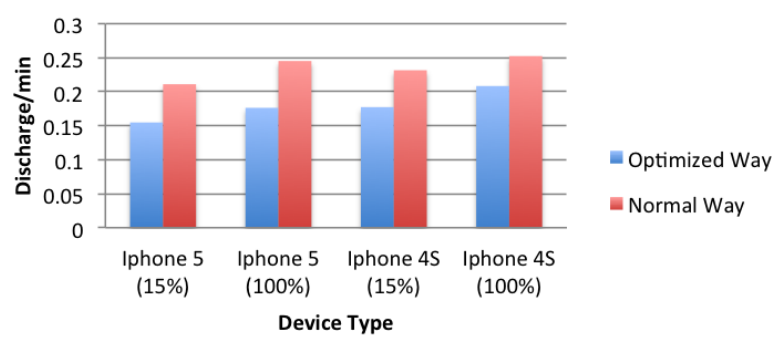

Fig 5: OpenGL drawing with caching mechanism

\subsection{Video Play Comparison: Hardware vs. Software Decoder}

From the power consumption calculation, it is found that hardware decoding lead to glitches in the video and cannot support many file types compared to software decoding. But software decoding requires much power. So to optimize power consumption for video play, PPAS approach uses hardware decoding for video play. Figure 4 shows the comparison. It demonstrates that hardware decoder consumes significant amount of less power than software decoder.

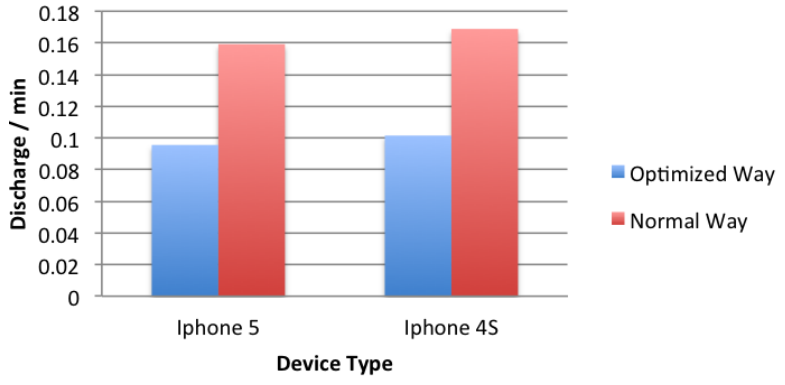

Fig 6: Backlight control with orientation

\subsection{OpenGL Drawing with Caching Mechanism}

To display OpenGL drawing with less power and high performance, OpenGL ES texture cache is used to get quick access to the raw pixels within an OpenGL ES texture. We take advantage of this to grab the pixels for an OpenGL ES rendering by using a frame buffer object with an attached texture which has been supplied from the texture cache. Therefore, it minimizes the unnecessary access to read the high resolution frame from main memory and saves power and makes the performance better. To compare power consumption, two applications are developed: one using cache mechanism and another without cache. Figure 5 shows the efficiency of proposed approach. The power discharge rate is low when we use caching mechanism to draw OpenGL views.

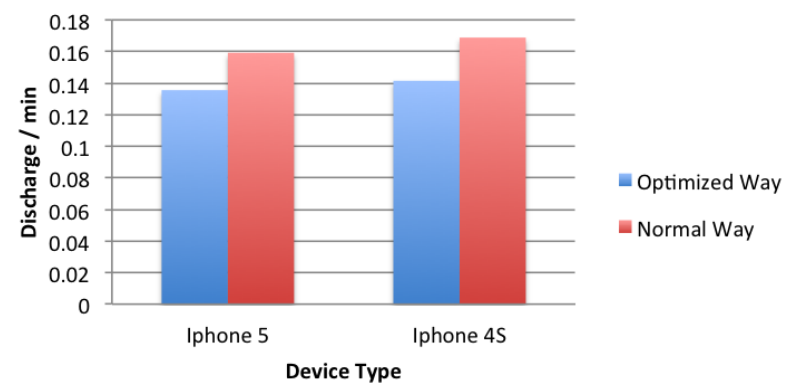

Fig 7: Backlight control with gyroscope

\subsection{Backlight Control with orientation or Gyroscope}

From data collection, it is found that backlight consumes a significant amount of power comparing to other components. Therefore, backlight can control perfectly by auto turn OFF and save power.

Usually when we use Smartphone it is not completely in vertical position. But often we keep Smartphone on table, floor or other vertical places when it's not used. So, this vertical position is detected using orientation or gyroscope to turn OFF power immediately and save significant amount of power. We develop an app with above mention technique and set sleep time to 15 seconds. It provides better solution for optimizing power consumption. The effectiveness of proposed approach is present in figure 6 and figure 7 . Both of the figure shows that if we use backlight with orientation or gyroscope mechanism, we can save power which will lengthen battery life. 


\subsection{Proper Use of Bluetooth}

Analyzing power consumption data of Bluetooth with some scenarios, it is found that discovery time and trying to pair time of Bluetooth with other devices plays an impact on power consumption. Most of the times we forget to switch OFF our Bluetooth option when it's not necessary. In proposed approach, it sets some time to turn OFF Bluetooth when it's not in pair state. The comparison of optimized approach with normal approach is shown in figure 8 .

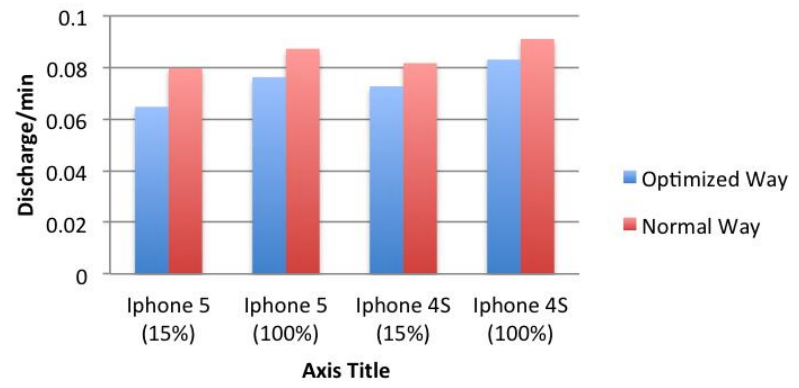

Fig 8: Proper use of Bluetooth

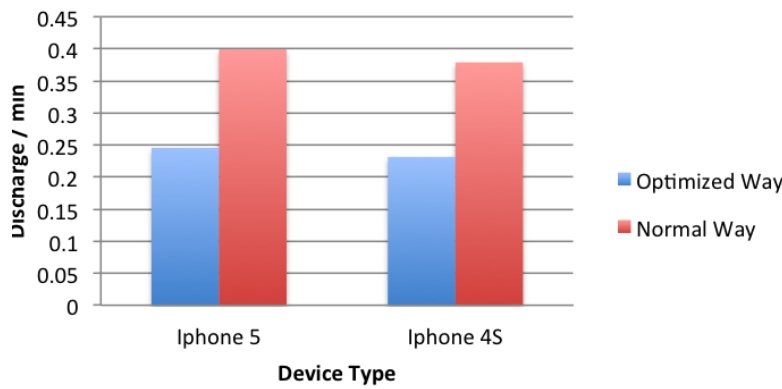

Fig 9: Background task handling when app switch frequently

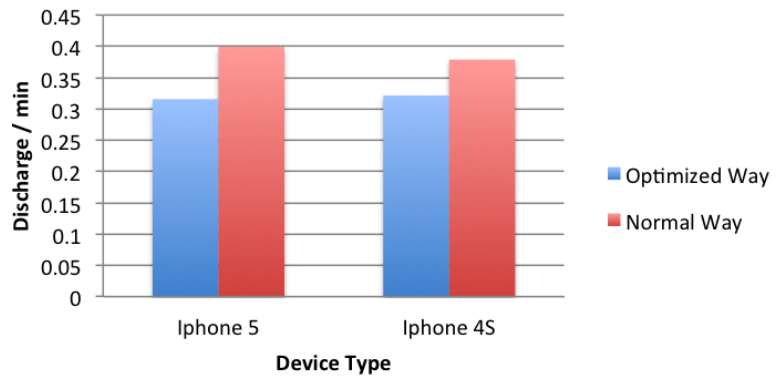

Fig 10: Background task handling when app switch rare

\subsection{Background Task Handling}

Often we don't consider background task but it cumulatively may drain the battery very quickly. PPAS avoids this power consumption by manually stop those task which are not necessary to run in background. To compare the output, we develop an app with the above mentioned technique and run the application for long time to collect data. While comparing, two cases has been considered: one is when app switch frequently and another is when app switch rare. Figure 9 and figure 10 show the robustness and effectiveness of our proposed optimized way.

\section{CONCLUSION}

This paper presents the design of PPAS method which can be used to analyze and optimize the energy consumption of Smartphone. We performed a details analysis of energy consumption of iPhone considering all major hardware components. We also focused on the major factors related to the drain of Smartphone battery life. Some effective techniques are also discussed which will help to lengthen the battery life of Smartphone. Experiments on different scenarios show that the proposed optimized techniques improve performance and saves battery with compared to the normal approach. This work will give both the Smartphone users and developers a good knowledge of the power usage in different scenarios. There are number of directions where researchers can contribute. This research can be applied for analysis power consumption of upcoming functionalities and latest technologies like LTE However, the power consumption analysis is done from application layer only, kernel or OS layer is not considered. We hope to improve our method so that it can do power consumption analysis both in application layer and OS layer.

\section{ACKNOWLEDGEMENTS}

Department of Computer Science and Engineering, University of Dhaka had given sufficient support and helps to complete this research by providing us lab facilities and research journals.

\section{REFERENCES}

[1] W. Browsing. http://en.wikipedia.org/wiki/web_browser. (accessed 10 Jan 2014)

[2] Falaki, H; Mahajan, R; Kandula, S; Lymberopoulos, D; Givondan, R; \& Estrin, D. 2010. Diversity in smartphone usage. In proceedings of the $8^{\text {th }}$ International conference on Mobile System, Applications and Services. 179-194.

[3] Carroll, A., Heiser, G., 2010. An analysis of power consumption in a Smartphone. In proceedings of the 2010 USENIX Conference on USENIX Annual Technical Conference.

[4] Crk, I., Albinali, F., Gniady, C., \& Hartman, J. 2009. Understanding energy consumption of sensor enabled applications on mobile phones. In Engineering in Medicine and Biology Society, 2009. EMBC 2009. Annual International Conference of the IEEE. pp. 68856888 .

[5] Ding, F., Xia, F., Zhang, W., Zhao, X., \& Ma, C. 2011. Monitoring energy consumption of Smartphone. In Internet of Things (iThings/CPSCom), 2011 International Conference on and $4^{\text {th }}$ International Conference on Cyber, Physical and Social Computing. 610-613. IEEE.

[6] Ogawa, T., Ito. K., \& Matsushima, K. 2013. Hardware Platform Supporting Smartphones. Fujitsu Sci. Tech. J. 49(2). 231-237.

[7] https://www.apple.com/iphone-5s/specs. (accessed 8 Dec 2014)

[8] https://www.apple.com/iphone-4s/specs. (accessed 8 Dec 2014) 